\title{
Study on the Self-Growth of the Internet Communication System
}

\author{
Yun-Feng Xia
}

stevenxia_bm@163.com, School of Publishing, Communication \& Management, Beijing Institute of Graphic Communication, Daxing, Beijing

\begin{abstract}
In this essay, I mainly discuss the self-organizing property of the Internet communication system. As an important part of the human information communication system, Internet communication has progressively become a system with large scale, complex constructions, and perfect functions through the complicated interactions among many sub-systems and their elements. In this process, which is mainly promoted by Internet users, the self-growth of the Internet communication system not only increases the amount of its sub-systems and its elements, it also strengthens its constructions and functions. At the same time, the self-growth of the Internet communication system indicates that the system is an evolving system with self-organizing property.
\end{abstract}

Keywords: Internet, Internet communication, Internet communication system, self-organization, self-growth

Acknowledgement: I wish to thank Jing-Shan Wang, who is an associate professor at the School of Publishing, Communication \& Management, Beijing Institute of Graphic Communication, and Zong-Rong Li, who is a professor of Huazhong University of Science and Technology, for their inspiring suggestions. This work did benefit from the discussion with Professor Wang and Professor Li.

Communication has always been one of the most important human activities. Forced by the pressure of living, people need to continuously acquire the information about environmental change. Meanwhile, for their own development, people also need to acquire, accumulate and share knowledge. All these needs can be met through communication activities. In a sense, we can say that the history of human is the history of communication evolution. In accordance with the development of mediums, we can divide the history of communication development into four stages, that is, oral communication, written communication, printing communication and electronic communication (Guo, 1999, pp. 28-34). The invention of computer brought us into the Internet communication age. Communication through network is the most important revolution in the human communication history. Internet communication possesses almost all advantages of previous human communication means, making both the scope and efficiency of communication have come a long way.

\section{Introduction to the Internet Communication System}

To explore the Internet communication, firstly, it is necessary to define related concepts. Many scholars have interpreted the definition of "communication" from various angles. In Chinese academia, scholars commonly accept the following viewpoint: "so-called communication, that is, the transmission of social information or the run of social information system" (Guo, 1999, pp. 1-6). This means the fundamental purpose of communication is to transmit information; information is the content of communication. Communication only refers to human communication from the narrow sense; the generalized one includes all the phenomena of transporting information in the whole nature. As for "network", in this article, it primarily refers to the world's largest computer networkInternet. Through linking with each other in the world, it has become the largest information system in human history.

Accordingly, we can define the Internet communication as the activities of disseminating human information (including news, knowledge, etc) through the computer network (Baidu, 2010). Namely, the action and process of human use computer network to communicate or transmit information 
(Wang, Shi and Ding, 2001). As a new form of human communication, Internet communication is the landmark of human communication revolution.

\subsection{Systemic Property of Internet Communication}

\subsubsection{Internet Communication is a Dynamic System, which is Evolved Through Self- Organizing}

Although the word "system" is often used in social life and academic fields, different people often give it different meanings in different places. In general, system is considered as "an organic whole with several interrelating and interacting elements and specific functions" (Wang, 1986, pp. 401).

According to this definition, the Internet communication is possessed of obvious systemicness. Internet communication is composited of multiple elements, like the network users and network information resources. The presence and arrangement of these elements is not random. Through complex interactions, the complicated relationship among these elements was formed. In this process, the Internet communication presents the characteristic of "1+1=2", that is, the emergent property, such as the formation of network culture (Sun \& Wei, 2005). The Internet communication will have stable macro-structure in a given time through these interactions. In addition, the Internet communication also has a series of functions. Among these, the most important one is to satisfy the users' information needs.

Based on the above analysis, it is nature to say that the Internet communication possesses systemic property; moreover, it is also a constantly evolving system. The macro-structure and functions of the Internet communication are relatively stable in a given time, but its micro-subsystems and their elements are changeable, such as the increase of the quantity of the network users. Meanwhile, complex interactions are existed among the subsystems and their elements. These changes and interactions promote the evolution of the system's scale, structures and functions, turning it into a dynamic information system, which is evolving constantly.

The Internet communication system reflects self-organizing property in the process of its evolution. Self-organization is the opposite of the term "organized". "If in the process of a system obtain the structure of space, time or function, there are no specific outside interventions, and we can say the system is a self-organizing system. The term 'specific' means the outside acts on the system through a non-specific way rather than exerts the structure and function on the system" (Haken, 1988, pp. 29). As a self-organizing system, network communication is a dynamically order system, its elements, structures and functions are changing constantly. However, in a given time, some aspects of the Internet communication system, such as the way of users attain the information, have the relative stability. This relative stability makes the Internet communication system have dynamically stable structures, which play an important role in maintaining the system's functions. Meanwhile, as an open system, the Internet communication system constantly exchanges material, energy and information with the outside, namely, the social system. For example, in the BBS, network users would like to discuss some things or people, the discussion will exert some impact on these things and people. Seen from a microcosmic angle, many elements of the Internet communication system are organized; but, to see from a macroscopic perspective, there is no "macrocontrol center" in the system, its structures and functions are formed by the interactions among the multiple subsystems and their elements. The above analysis indicates that the Internet communication system is a self-organizing system.

\subsubsection{Factors Constituting Internet Communication System}

As a new form of human communication, Internet communication has its own personality and commonality of human communication. Therefore, we can use some research achievements attained in human communication field to analyze the Internet communication. In this article, I will use the Lasswell's "5W" (that is, "Who", "Says What", "In Which Channel", "To whom" \&" With what effects") communication model to analyze the elements of the Internet communication. In 1948, American scholar Harold D.Lasswell put forward the five basic elements constituting communica- 
tion process and ordered them according to a certain structural arrangement in one of his articles. This is the famous " $5 \mathrm{~W}$ " communication model (see Figure 1 ).

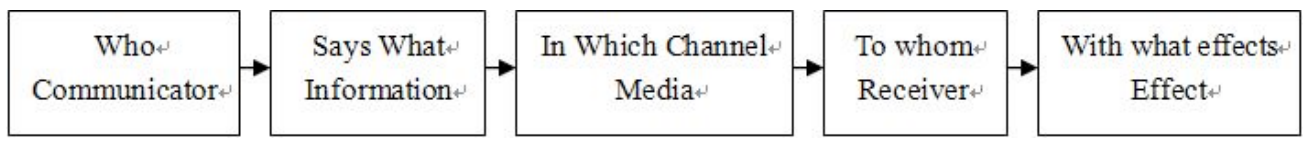

Figure 1: The Lasswell's “5W” communication model (McQuail, D. \& Windahl, S., 1981, pp. 10)

The five elements are communicator, information, media, receiver and effect. According to the "5W" communication model, we can simplify the Internet communication into four parts, namely, the Internet users, the Internet information resources, the Internet communication channels and the Internet communication effect.

The network users are all the participants of Internet communication activities, including the producers, providers and consumers of the network information and services. They are the starting point and termination of the Internet communication. They also play an intermediary role in the system. In the system, the role of the communicator and receiver is not fixed because feedbacks and interactions are very easy.

All kinds of resources we can use through the computer network are the Internet information resources (Baidu, 2009). The Internet resources are the content of network communication and present the following characteristic: digital storage, enormous quantity, complicated information source and so on.

Internet communication channels, which are composed of the network connecting devices, network transport protocols, network transmission mediums, etc, are the paths and equipments to transfer and store network information. The Internet communication channels prepare the prerequisite and foundation for the Internet communication.

The process of network users attain, digest, absorb and reuse the Internet information resources has a certain impact on the individual and society; we can call these effects the Internet communication effect (Meng, 2007). These effects can be divided into positive effect and negative effect. The former one is good for the individual and the society, the latter one is the opposite of the former one.

\section{Self-Growing Property of the Internet Communication System}

The birth of a system does not mean the end of its evolution. After a system being arouse, its own structures and functions are needed to improve and optimize constantly. As a system, in order to survive and develop, Internet communication also needs to undergo this process. In this process, the system's macroscopic structures and functions will keep stable; this is the self-growth of the Internet communication system.

\subsection{Performance of the Internet Communication System's Self-Growth}

Self-growth describes the evolution of the system's condition formed by the self-organization with time from a macroscopic angle. In the process of self-growth, only the "volume" of system was increased, its shape, nature and character are unchangeable. Its structures and functions also keep invariable (Sun \& Lin, 2004). The phenomenon of self-growth is widespread, such as the evolution of the eco-system. Self-growth is a quantitative change and "gradual" process, which provides the possibility for the system's "qualitative change".

In the Internet communication system, the phenomenon of self-growth is also common. Among them, the increase of the quantity of the network users and web sites is the most obvious phenomenon of self-growth. Take Chinese network users as an example. In 1997, only 20million Internet users were in China. But the number reached to 2,200 million in 2001. This means the number grew full 100 times in 3 years (Chinanews, 2001). In 2009, this figure amounted to more than 384 million (CRI Online, 2010). At the same time, the amount of global web sites also increased rapidly. 
Relevant surveys showed that in 2008, the total number of global web sites was 186.7 million (ChinaZ, 2009). And by 2009, this number increased to 234 million (ChinaZ, 2010). The increase of the number of network users and web sites are both caused by self-growth. Based on their own needs rather than others' commands, network users chose to use the Internet. The increase of the quantity of web sites is also a self-organizing process. A specific site may be organized. But network users have considerable rights to choose which type of web site will be established and which kind of information resource will be introduced into the network. He or she can also decide how to link with other sites. As a result, this is also a self-organizing process.

At the same time, the Internet information resources are also increased rapidly. When the Internet was invented, very limited information resources were communicated in the network. Nowadays, the total number of the Internet information resources can not be precisely estimated. By now, Internet information resources have been covered almost all social aspects, including news, education and medical field. By the same token, this process also reflected the self-growing property of the Internet communication system; because the amount and type of information resources introduced into the system is not determined by the orders from a unified control center.

Of course, these are only parts of self-growing phenomenon of the Internet communication system. In the system, other elements, like the network hosts and hyperlinks, are all growing constantly. The self-growth not only expands the system's "volume", but also greatly enhances its vitality.

\subsection{Main Impetus to the Internet Communication System's Self-Growth}

In order to sustain continuous development, any system shall hold enough impetus to prolong the process of its development. For example, the growth of the human body's development needs material and energy from the outside continuously. So the same thing is with the Internet communication system. Take the individual role each element plays in the process of the system' development into consideration, we can come to a conclusion that the Internet user, the most active element, is the main self-growing impetus to the Internet communication system. The roles of the Internet users can be reflected in the following aspects: the growth of the quantity of the Internet users, the enrichment of the Internet information resources and the development of the Internet communication technology. The growth of the quantity of the Internet users, the result of their free choices, embodies the initiative of the Internet users. The enrichment of the Internet information resources and the development of the Internet communication technology also need the Internet users. Internet users willingly post all sorts of information from the outside to the Internet communication system continuously. At the same time, they may produce new information resources through digesting the present resources of the system (Xu, 2009). The Internet users also prompt the coming of new generation of the Internet communication technology. The Internet communication system will be developed due to the invention of the new Internet communication technology and the modified technology of the present.

In some sense, the reason why the Internet communication system can develop from a computer network to an "active" human-computer system is the participation of the Internet users. In the system, "person" is the main player and in the decisive position (Sun \& Wei, 2005). Using the Internet is based on the different demands of the Internet users, and the demands also become the original motive of the system's development. The enrichment of the Internet information resources, the development of the Internet technology, and even perfection and optimization of the structures and functions of the Internet communication system all come to meet the demands of the Internet users. Nowadays, "person" has been deeply "embedded" into the Internet communication system and becomes the indispensable part of it.

The motive source of the Internet communication system' self-growth well testifies why the Internet communication system should be an open system. In the case that the Internet communication system is a closed system, and has no exchange of material, energy and information with the outside, there is no growth of quantity in terms of Internet users and Internet information resources. That is because the growth of Internet users means that the "persons" from the social system enter 
the Internet communication system continuously. Without the participation of the "persons" from the social system, the growth of quantity of the Internet users is meaningless. Besides, the enrichment of the Internet information resources is mainly due to the outside environment although some parts are from the new Internet information resources produced through digesting the present Internet information resources. Once the Internet communication system is separated from the outside, this part of information resources will not be appeared in the Internet communication system.

\subsection{Restricted Factors of the Internet Communication System's Self-Growth}

The interactions between various elements bring about the self-growth of the Internet communication system; therefore, the self-growing process is also affected by these elements.

Internet users are the main part of the Internet communication system and the most important promoter of the self-growth. Whether they use the network or not will have great impact on the expansion of the system's scale. Based on their own will, the Internet users would like to build various types of web sites and introduce information resources into the Internet communication system. These activities have been the main promoter of increasing the quantity of the web sites and the network information resources. Meanwhile, for increasing the click ratio and gaining "eyeball economy", each site must carefully analyze the specific needs of the Internet users and provide corresponding information resources and services for them. This will improve the quality of network services. From the above, we can see that the number of network users, their willingness to participate, and their demands will largely determine the evolving direction of the Internet communication system.

Internet communication channels, as the transmission channels and storage space of the Internet information resources, lay the foundation for network communication. Without unimpeded channels and adequate storage rooms, the network resources and the interactions among network users no longer exist. In December 2006, many Chinese network users can not access to overseas web sites because of the interruption of multiple international submarine cables caused by the earthquake in Taiwan (Sina, 2006). This has fully demonstrated that the Internet communication channels play an important role in the Internet communication system.

In addition, the Internet communication system, as a part of the social system, is deeply affected by the environmental change of social system. Based on management's needs, government draws up relevant laws and regulations, and employs some administrative measures (Wang, Yao and $\mathrm{Li}$, 2009). These laws, regulations and administrative measures, no matter act on network users or Internet information resources, will exert a certain influence on the self-growth of the Internet communication system. They also influence the direction and speed of the system's development.

\subsection{Significance of the Internet Communication System's Self-Growth}

\subsubsection{Improving the Structures and Functions of the Internet Communication System}

The self-growth of the Internet communication system is the quantitative changing process of the system and its elements. In this process, the system only increased its "volume", its structures and functions were unchangeable. But, by self-growth, the system's structures and functions were selfimproved and self-optimized. In the revolving process, the increase of the quantity of the Internet users and enrichment of the Internet resources both will enhance the system's complexity; the complexity of interactions among related subsystems and their elements is increased relatively. In order to adapt to the new environment, the system's structures and functions have to evolve continuously. This always makes these structures and functions more perfect and improved.

In a word, the self-growth of the Internet communication system promotes the improvement of its structures and functions. Take the development of Internet search engine as an example. Originally, Internet search engine was a tool that can optimize relevant web pages and provided the optimized results for users (Baidu, 2010); the services supplied by it were very limited. But with the increase of Internet users and the diversification of demand, combined with the enrichment of Internet information resources, nowadays, the types of service provided by the search engine has 
already covered almost all aspects of social and private life. For example, Google search engine has already supplied a dozen services, including Web Search, Book Search and Scholar search (see Figure 2).

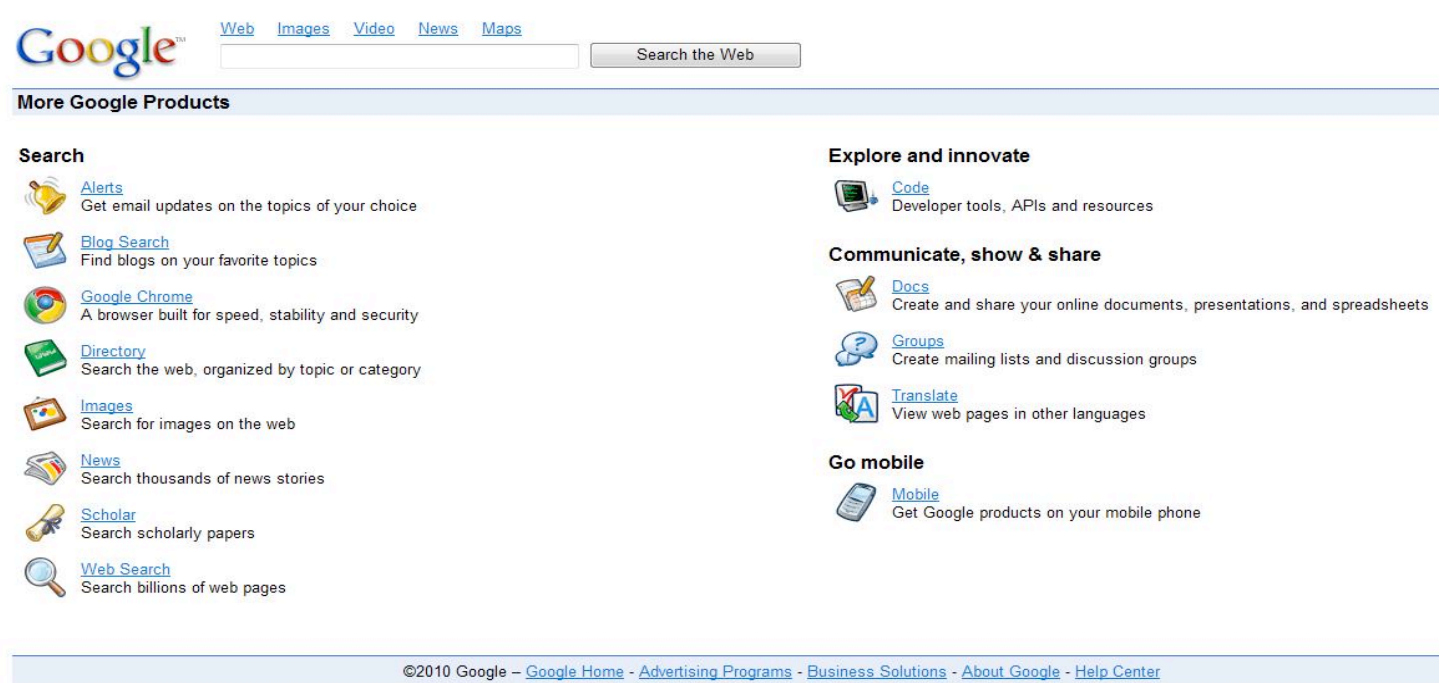

Figure 2: List of the functions provided by Google

\subsubsection{Showing the Internet Communication System is a Self-Organizing System}

The self-growth of the Internet communication system is an important reflection of the system's evolution, which is advanced by self-organization. In the Internet communication system, the changeable elements and the macroscopic "qualitative change" caused by the "quantitative change" of these elements both show that the system is an evolving system. Meanwhile, the change of elements and the "qualitative change" of the system are self-completed through the interactions among many subsystems and their elements. In this process, there is no unified control center; the direction of their evolution is not set in advance.

The self-growth of the Internet communication system has a great effect on its self-organizing process. As a process of "quantitative change", the growth of Internet communication system lays the foundation for its qualitative change. After a certain limit being broken, the system will derive new structures and functions from the previous one. Take the web search engine for example. With enrichment of Internet information resources, the efficiency of obtaining useful information was dropped constantly; this brings about imbalance between the supply and the demand. Internet search engine was invented in this context and greatly improved the efficiency through optimalizing the relative web pages (Su \& Ou, 2008). We can come to a conclusion that the self-growth of the Internet communication system is a process of accumulating "energy" that is needed by the selforganization. This "energy" is the occasion of the self-organizing revolution; it also continuously provides enough power for the system's development.

\section{Conclusions}

As a new form of human communication, Internet communication has evolved from the originally pure computer network to a complicated system integrating human and machines via the interactions between multiple systems and their elements. During its evolution, the Internet communication system exhibited a characteristic of self-growth. Both the increase in the number of network users and the expansion of Internet communication resources are the reflection of this characteristic. Through self-growth, the Internet communication system has not only expanded its size but also improved and optimized its structures and functions continuously. The system is therefore in a process of continuous self-improvement. 
As a system capable of self-growth, the Internet communication system develops based on the premise that its multiple elements are able to freely evolve and interact with each other. A good environment for the evolution and interactions is needed both inside and outside of the Internet communication system. Inside the system, the network communication channels must be through all the time. Moreover, new communication technologies must be introduced into the Internet communication system to speed up the efficiency of evolution and interactions. Outside of the system, the social system should also create conditions for the evolution and interactions between these elements. To meet the need for management, the government will exercise its influence on the Internet communication system by imposing laws, regulations and administrative orders. Such influence, however, should be limited within a range. If excessively high barriers are set for network users' access to networks or for external information's access to networks, the evolution of the Internet communication system will slow down and even come to a halt. Only in a good environment, can the many elements of the system achieve evolution of their own and interactions with each other, eventually prompting the evolution of the Internet communication system.

The self-growth of the Internet communication system shows that a complicated structure and function can be evolved from a simple one through self-growth (Yin \& Zhou, 2005). Single LANs (local area network) constituting the Internet are not complicated at all in structures and functions and can be easily defined. But, once these LANs are linked with each other, their structures and functions will become extremely complicated. The reason for this phenomenon is that although self-growth is a process of quantitative change, this quantitative change will produce a qualitative change at some point, which will create new structures and additional functions for the system. The new structure and functions will result in new complexity.

\section{References}

Baidu. (2009). Internet Information Resource. Retrieved May 6, 2010 from http://baike.baidu.com/view/1356256.htm?fr=ala0_1

Baidu. (2010). Internet Communication. Retrieved May 6, 2010 from http://baike.baidu.com/view/967588.htm?fr=ala0_1

Baidu. (2010). Search Engine. Retrieved May 6, 2010 from http://baike.baidu.com/view/1154.htm?fr=ala0 11

Chinanews. (2001). Experts predicted that after 5 years China will have the largest network scale in the world. Retrieved May 6, 2010 from http://www.chinanews.com.cn/2001-10-19/26/131980.html

ChinaZ. (2010). The number of global Internet sites reached 234 million, the kind of .com accounted for 81.8 million. Retrieved May 6, 2010 from http://www.chinaz.com/Webmaster/report/01261050052010.html

ChinaZ. (2009). The number of Internet sites reached 187 million, but less than $4 \%$ of them were active. Retrieved May 6 , 2010 from http://www.chinaz.com/Webmaster/Club/0105593452009.html

Chun-Hua, Y. \& Xi-Ping, Z. (2005). Discussion on Internet Complication. Information Science, 23(4), 568-572.

CRI Online. (2010). The number of Chinese netizens reached 384 million, ranking first in the world. Retrieved May 6, 2010 from http://gb.cri.cn/27824/2010/02/03/3365s2749528.htm

Dong-Chuan, S. \& Bin-Yong, W. (2005). Systematic Analysis of the Peculiarity of Internet. Journal of Systemic Dialectics, 13(2), 49-52.

Dong-Chuan, S. \& Fu-Yong, L. (2004). Introduction to Systems Engineering. Beijing: Tsinghua University Press.

Haken, H. (1988). Information and Self-Organization. Chengdu/Sichuan: Sichuan Education Publishing House.

Jia-Ning, X. (2009). Study on the Mechanism of Internet Information Self-organizing Based on Web2.0. Journal of intelligence, 28(6), 139-142.

McQuail, D. \& Windahl, S. (1981). Communication Models. London: Longman Group Ltd..

Qing-Guang, G. (1999). Introduction to Communication Studies. Beijing: China Renmin University Press.

Qing-Lan, M. (2007). Analysis of the Effect of Internet Information Communication. Researches in Library Science, (11), 8588.

Rui-Zhu, S. \& Yang-Jian, O. (2008). On the Self-Organization and Re-organization of Network Information Resource. Information Science, 26(1), 60-63.

Sina. (2006). Exclusive: Telecom said the disruption of many submarine cables caused international network failure. Retrieved May 6, 2010 from http://tech.sina.com.cn/i/2006-12-27/10491308770.shtml

Xia, W., Si-Cong, Y. \& Da, L. (2009). Social Management on Internet Communication. Journal of Hubei University of Police, (1), 125-128.

Yu-Tian, W. (1986). Cybernetics, Information Theory, Systems Science and Philosophy. Beijing: China Renmin University Press.

Zhong-Yi, W., Liang, S. \& Yi-Dai, D. (2001). Internet Communication: Principle and Practice. Hefei/Anhui: University of Science and Technology of China Press. 


\section{About the Author}

\section{Yun-Feng Xia}

is from China and graduated from Beijing Institute of Graphic Communication and received the degree of Bachelor of Printing Engineering in 2007. Yun-Feng is currently a graduate student at the School of Publishing, Communication \& Management, Beijing Institute of Graphic Communication, researching digital communication. 